\title{
Monitoring Tumor Therapy
}

This unit presents the preferred set of MR sequences for imaging the brain following the institution of chemotherapy, radiation therapy, or surgery for brain tumors (see Basic Protocol 1) and specific modifications will be discussed where necessary (see Basic Protocol 2). The sequences described in this unit are based on the authors' experience with a $1.5 \mathrm{~T}$ scanner (Echospeed GE Medical Systems), but can be expected to be equally applicable to other field strengths and scanners from other manufacturers. Sequences 1 to 5 comprise the preferred Basic Protocol 1.

\section{FOLLOW-UP POST SURGERY}

One of the most important issues for initial post-operative MRI of the brain is that it should be performed within 24 to $48 \mathrm{hr}$ of surgery to assess for any enhancing residual tumor. Noncontrast $T_{1}$-weighted images are important to obtain in the same imaging plane as the post-contrast $T_{1}$-weighted images to assess for residual tumor and not mistake blood products for enhancing tumor. Sequences 1 to 5 comprise the preferred protocol.

Table A3.5.1 lists the hardware necessary to perform the procedure, along with appropriate parameters.

NOTE: Be sure that technologists and nurses have immediate access to any equipment such as crash carts or oxygen that may be necessary in the event of an emergency. Reactions to contrast agents are rare, but the resources are necessary.

\section{Materials}

Intravenous contrast agent (e.g., Magnevist, Omniscan, Prohance)

Normal saline $(0.9 \% \mathrm{NaCl})$, sterile

\section{Setup patient and equipment}

1. Interview (screen) the patient to ensure that he or she has no contraindications to the MRI examination, such as a cardiac pacemaker or other implants containing ferromagnetic materials. Question the patient regarding any health conditions that may require the presence of specific emergency equipment during the scanning procedure, or necessitate any other precautions.

Generally standard screening forms are used for all patients scanned in a magnetic resonance system.

The presence of ferromagnetic metals may be a health hazard to the patient when he or she is inside the magnet, and will also affect the imaging. If in doubt as to the exact composition of the items, it is best to exclude patients with any metal implants; see Shellock (1996) for discussion of what implants may be safely scanned using magnetic resonance.

Table A3.5.1 Equipment Parameters for Imaging Brain Tumors

\begin{tabular}{ll}
\hline Coil type & Quadrature head coil \\
Gradient coil strength & $25 \mathrm{mT} / \mathrm{m}$ (or whatever the system permits) \\
Cardiac gating & No \\
Peripheral gating & For safety only \\
Respiratory gating & No \\
Respirator & If required by patient \\
Oxygen & If required by patient \\
Motion cushions & No
\end{tabular}

Contributed by Annette O. Nusbaum and Scott W. Atlas

Current Protocols in Magnetic Resonance Imaging (2001) A3.5.1-A3.5.7

Copyright $@ 2001$ by John Wiley \& Sons, Inc.

BASIC

PROTOCOL 1

Cerebral Neoplastic Disease

A3.5.1

Supplement 1 
Patients may be accompanied into the magnet room by a friend or family member, who can sit in the room during the scan and comfort the patient as needed. This companion must be screened as well to ensure the absence of loose metal objects on the body or clothing.

2. If the procedure is a research protocol, have the patient sign any necessary consent form.

3. Have the patient remove all jewelry and change into a gown to eliminate any metal that might be found in clothing.

4. Have the patient wash off any mascara and other makeup to avoid local tissue heating and image artifacts.

5. Inform the patient about what will occur during the procedure, what he or she will experience in the magnet, and how to behave, including the following:

a. If earphones or headphones are used to protect the ears from the loud sounds produced by the gradients, the patient will be asked to wear these, but will be able to communicate with you at any time during the imaging.

b. The patient will be given a safety squeeze-bulb or similar equipment to request assistance at any time (demonstrate how this works).

c. For good results the patient should not talk, and should avoid or minimize swallowing or other movement, during each scan-i.e., as long as the banging sounds continue. Between scans, talking and swallowing are allowed in most cases, but should be avoided when comparative positional studies are being performed; the patient will be informed when this is the case.

d. Nevertheless, the patient may call out at any time if he or she feels it necessary.

6. Have the patient mount onto the table in the supine position. Either before or right after the patient lies down, set up any triggering devices or other monitoring equipment that is to be used.

7. Center the patient in the head coil at the region where the key information is desired. Make sure the head and neck are constrained to prevent motion.

Generally the patient's head is fixed so that the head is horizontal (not tilted) and the neck and head lie along the axis of the patient table.

8. If needed, place a pillow or other support under the knees to make the patient more comfortable.

9. Use the centering light to position the patient (centered on the nasion) and put him or her into the center of the magnet.

Once this step has been performed, so long as the patient does not move on the table, the table itself can be moved and then replaced in the same position as before without jeopardizing the positioning of one scan relative to another.

10. If the patient is unable to hold still, provide an appropriate sedative.

\section{Sequence 1: Localizer}

11. Run sequence 1 according to Table A3.5.2.

\section{Sequence 2: $T_{1}$-weighted scan}

12. Run sequence 2 according to Table A3.5.3.

\section{Sequence 3: $T_{2}$-weighted scan}

13. Run sequence 3 according to Table A3.5.4.

Sequence 4: Fluid attenuated inversion recovery (FLAIR) scan 
Table A3.5.2 Primary Clinical Imaging Parameters for Sequence 1 ( $T_{1}$-Weighted)

\begin{tabular}{|c|c|}
\hline Patient position & Supine \\
\hline Scan type & Spin echo \\
\hline Imaging plane (orientation) & Sagittal \\
\hline Central slice or volume center & Laser light centered on nasion \\
\hline Echo time $\left(T_{\mathrm{E}}\right)$ & $\begin{array}{l}11 \text { msec (or select "minimum } \\
\text { full" echo time) }\end{array}$ \\
\hline Receiver bandwidth (RBW) & $10 \mathrm{kHz}$ \\
\hline Repeat time $\left(T_{\mathrm{R}}\right)$ & $500 \mathrm{msec}$ \\
\hline Flip angle (FA) & $90^{\circ}$ \\
\hline Fields of view $\left(\mathrm{FOV}_{\mathrm{x}}, \mathrm{FOV}_{\mathrm{y}}\right)$ & $240 \mathrm{~mm}, 240 \mathrm{~mm}$ \\
\hline Resolution $(\Delta x, \Delta y)$ & $0.94 \mathrm{~mm}, 1.25 \mathrm{~mm}$ \\
\hline Number of data points collected $\left(N_{\mathrm{x}}, N_{\mathrm{y}}\right)$ & 256,192 \\
\hline Slice thickness $(\Delta z)$ & $5 \mathrm{~mm}$ \\
\hline Number of slices & $\begin{array}{l}20 \text { or as many as needed to cover } \\
\text { the region of interest }\end{array}$ \\
\hline Slice gap & $2 \mathrm{~mm}$ \\
\hline Number of acquisitions $\left(N_{\mathrm{acq}}\right)$ & 1 \\
\hline Swap read and phase encoding & No \\
\hline Saturation pulses & Not applicable \\
\hline Scan time & $1 \mathrm{~min}, 36 \mathrm{sec}$ \\
\hline
\end{tabular}

Table A3.5.3 Primary Clinical Imaging Parameters for Sequence 2 ( $T_{1}$-Weighted)

\begin{tabular}{ll}
\hline Patient position & Supine \\
Scan type & Spin echo \\
Imaging plane (orientation) & Transverse \\
Central slice or volume center & Laser light centered on nasion \\
Echo time $\left(T_{\mathrm{E}}\right)$ & $11 \mathrm{msec}$ (or select "minimum \\
& full” echo time) \\
Receiver bandwidth (RBW) & $10 \mathrm{kHz}$ \\
Repeat time $\left(T_{\mathrm{R}}\right)$ & $500 \mathrm{msec}$ \\
Flip angle $(\mathrm{FA})$ & $90^{\circ}$ \\
Fields of view $\left(\mathrm{FOV}, \mathrm{x}, \mathrm{FOV}_{\mathrm{y}}\right)$ & $240 \mathrm{~mm}, 240 \mathrm{~mm}$ \\
Resolution $(\Delta x, \Delta y)$ & $0.94 \mathrm{~mm}, 1.25 \mathrm{~mm}$ \\
Number of data points collected $\left(N_{\mathrm{x}}, N_{\mathrm{y}}\right)$ & 256,192 \\
Slice thickness $(\Delta z)$ & $5 \mathrm{~mm}$ \\
Number of slices & 20 or as many as needed to cover \\
& the region of interest \\
Slice gap & $2.5 \mathrm{~mm}$ \\
Number of acquisitions $\left(N_{\mathrm{acq}}\right)$ & 1 \\
Swap read and phase encoding & Yes \\
Saturation pulses & Not applicable \\
Scan time & $1 \mathrm{~min}, 36$ sec \\
\hline
\end{tabular}

Cerebral 
Table A3.5.4 Primary Clinical Imaging Parameters for Sequence 3

( $T_{2}$-Weighted)

Patient position

Scan type

Imaging plane (orientation)

Central slice or volume center

Echo time $\left(T_{\mathrm{E}}\right)$

Receiver bandwidth (RBW)

Echo train length (ETL)

Repeat time $\left(T_{\mathrm{R}}\right)$

Flip angle (FA)

Fields of view $\left(\mathrm{FOV}_{\mathrm{x}}, \mathrm{FOV}_{\mathrm{y}}\right)$

Resolution $(\Delta x, \Delta y)$

Number of data points collected $\left(N_{\mathrm{x}}, N_{\mathrm{y}}\right)$

Slice thickness $(\Delta z)$

Number of slices

Slice gap

Number of acquisitions $\left(N_{\text {acq }}\right)$

Swap read and phase encoding

Saturation pulses

Scan time
Supine

Fast spin echo

Transverse

Laser light centered on nasion

$102 \mathrm{msec}$ (effective)

$16 \mathrm{kHz}$

8

$3600 \mathrm{msec}$

$90^{\circ}$

$240 \mathrm{~mm}, 240 \mathrm{~mm}$

$0.94 \mathrm{~mm}, 1.25 \mathrm{~mm}$

256, 192

$5 \mathrm{~mm}$

20 or as many as needed to cover the region of interest

$2.5 \mathrm{~mm}$

1

Yes

Not applicable

$1 \mathrm{~min}, 30 \mathrm{sec}$

Table A3.5.5 Primary Clinical Imaging Parameters for Sequence 4 (Fast FLAIR)

Patient position

Scan type

Imaging plane (orientation)

Central slice or volume center

Echo time $\left(T_{\mathrm{E}}\right)$

Receiver bandwidth (RBW)

Echo train length (ETL)

Repeat time $\left(T_{\mathrm{R}}\right)$

Inversion time $\left(T_{\mathrm{I}}\right)$

Flip angle (FA)

Fields of view $\left(\mathrm{FOV}_{\mathrm{x}}, \mathrm{FOV}_{\mathrm{y}}\right)$

Resolution $(\Delta x, \Delta y)$

Number of data points collected $\left(N_{\mathrm{x}}, N_{\mathrm{y}}\right)$

Slice thickness $(\Delta z)$

Number of slices

Slice gap

Number of acquisitions $\left(N_{\text {acq }}\right)$

Swap read and phase encoding

Saturation pulses

Scan time
Supine

Inversion recovery fast spin echo

Transverse

Laser light centered on nasion

$120 \mathrm{msec}$ (effective)

$16 \mathrm{kHz}$

8

$10,000 \mathrm{msec}$

$2200 \mathrm{msec}$

$180^{\circ}$

$240 \mathrm{~mm}, 240 \mathrm{~mm}$

$0.94 \mathrm{~mm}, 1.25 \mathrm{~mm}$

256, 192

$5 \mathrm{~mm}$

20 or as many as needed to cover the region of interest

$2.5 \mathrm{~mm}$

1

Yes

Not applicable $\sim 5 \mathrm{~min}$ 
Sequence 5: Post-contrast imaging

15. Remove the patient from the scanner. The patient should not move on the table. Establish an intravenous line from which the contrast agent can be injected, and attach this line securely to the patient so that movement into or out of the magnet will not pull at the patient's arm. Move the patient back into the scanner.

It is preferable to insert the line prior to imaging and to leave the patient in the magnet, with no intervening motion, between the scans run before contrast agent injection and those run after injection.

16. Leaving the patient in the magnet, inject the contrast agent, flush the line with $10 \mathrm{ml}$ saline.

A dose of $0.1 \mathrm{mmol} / \mathrm{kg}$ of contrast agent is usually given.

A delay in scanning may actually be beneficial when evaluating for metastases, which is one of the reasons we scan in multiple planes after intravenous contrast administration.

17. Acquire the post-contrast images using the same parameters as sequence $2\left(T_{1^{-}}\right.$ weighted).

In addition to the transverse plane, the coronal plane is routinely obtained, with the following changes to the parameters in Sequence 2: (a) flow compensation is on; (b) $T_{E}$ is 20 msec (prolonged due to flow compensation gradients); and (c) it is not necessary to swap read and phase encoding directions. In patients requiring sedation and in all pediatric patients, we also routinely obtain a third post-contrast plane of imaging (sagittal).

\section{RADIATION NECROSIS VERSUS TUMOR RECURRENCE}

Radiation necrosis and recurrent tumor are indistinguishable by conventional MR techniques and on the basis of paramagnetic enhancement, since both may appear mass like and enhance in most circumstances (Grossman et al., 1988). The time interval between radiation therapy and the MR scan is an essential part of making an intelligent presumptive diagnosis of either radiation necrosis or recurrent tumor (Atlas and Lavi, 1996). Several studies suggest that proton MR spectroscopy may be a potentially useful method for differentiating tumor recurrence from radiation necrosis (Taylor et al., 1996; Nelson et al., 1999; Ricci et al., 2000). Whole brain chemical-shift imaging (CSI) maps may also be potentially useful in the investigation of brain tumors by providing visual metabolite maps, rather than spectral data.

\section{Setup patient and equipment}

1. Use the same equipment and perform the same setup as for the previous method (see Basic Protocol 1).

2. Run sequences 1 to 5 (see Basic Protocol 1).

3. Localize the lesion on a transverse image (typically the post-contrast transverse $T_{1}$-weighted image).

The voxel placement requires physician input to ensure the correct region of interest is being studied. Two separate scans are performed: one with the voxel placed over the area in question (typically the enhancing portion of the lesion), the other scan is performed with the voxel placed in the contralateral hemisphere (in a "normal appearing" analogous area of the brain). The screen should always be saved to document placement of the voxel.

\section{Sequence 6: Proton $\left({ }^{1} H\right)$ MR Spectroscopy}

The proton brain exam (PROBE) on GE systems is an automated acquisition that is widely available and is performed using the single voxel technique $\left(8 \mathrm{~cm}^{3}\right)$. A high field system is required to obtain good spectra. There are 2 possible localization methods: STEAM

Cerebral 
(stimulated-echo acquisition mode) or PRESS (point-resolved spectroscopy). The sequence parameters can be set by the user $\left(T_{\mathrm{R}}, T_{\mathrm{E}}\right.$, voxel size). Short $T_{\mathrm{E}}$ spectroscopy has the advantage of providing more information, since more peaks are visible for analysis and detection of lipid-breakdown products is maximized. Long $T_{\mathrm{E}}$ spectra have less baseline distortion and are easier to quantify. Since there is a $\sqrt{2}$ decrease in the signal-to-noise ratio with STEAM sequences, the PRESS sequence is usually preferred. Spectra obtained following the administration of intravenous contrast may be altered (Sijens et al., 1997). In particular, studies have shown a mean loss of $15 \%$ of the peak area of choline-containing compounds without significant changes in creatine (Cre) or $\mathrm{N}$-acetyl aspartate (NAA) (Sijens et al., 1997). In routine clinical practice, the spectra are obtained following the administration of intravenous contrast and the voxel is placed over the solid (enhancing) portion of the lesion so that theoretically signal is acquired from viable tumor tissue.

4. Perform automated shimming and water suppression, center the voxel over the enhancing portion of the lesion, and run sequence 6 according to Table A3.5.6.

5. After completion of the scan, a spectrum will appear on the screen and the values for the spectrum should be saved.

6. Place the voxel in the contralateral hemisphere and repeat the scan.

7. After completion of the scan, another spectrum will appear on the screen and the values for the spectrum should be saved.

Table A3.5.6 Primary Clinical Imaging Parameters for Sequence $6\left({ }^{1} \mathrm{H}\right.$ MR Spectroscopy)

\begin{tabular}{ll}
\hline Patient position & Supine \\
Scan type & PROBE \\
Voxel Center & See text (steps 4 and 6) \\
Echo time $\left(T_{\mathrm{E}}\right)$ & $35 \mathrm{msec}$ and $144 \mathrm{msec}$ \\
Repeat time $\left(T_{\mathrm{R}}\right)$ & $2000 \mathrm{msec}$ \\
Flip angle $(\mathrm{FA})$ & $90^{\circ}$ \\
Voxel size $(\Delta x, \Delta y, \Delta z)$ & $2 \mathrm{~cm}, 2 \mathrm{~cm}, 2 \mathrm{~cm}$ \\
Number of acquisitions $\left(N_{\text {acq }}\right)$ & $8^{a}$ and $128^{b}$ \\
Saturation pulses & Water \\
Scan time & $\sim 5 \mathrm{~min}$ \\
\hline
\end{tabular}

${ }^{a}$ Without water suppression.

${ }^{b}$ With water suppression.

\section{COMMENTARY}

\section{Background Information}

Magnetic resonance imaging is a well-established tool in the evaluation of brain tumors by offering superb anatomical detail and tissue characterization of brain lesions. Conventional MRI techniques have limitations in the ability to discriminate between recurrent tumor and radiation necrosis as well as determining the exact limits of tumor extension. Postoperative patients being studied by MRI for evaluation of residual or recurrent tumor do not pose as difficult a problem as patients who receive radiation therapy. Contrast enhanced imaging is critical to the evaluation of post-therapy brain tumors. Proton MR spectroscopy may be a potentially useful tool for differentiating tumor recurrence from radiation necrosis (Taylor et al., 1996; Nelson et al., 1999).

\section{Critical Parameters and Troubleshooting}

A critical component of the MR examination is an adequate history of the therapeutic intervention instituted. Precontrast $T_{1^{-}}$ weighted images frequently demonstrate subacute blood products and need to be carefully 
compared with post-contrast images to assess for enhancing portions of the area of interest. In pediatric patients and other subjects requiring sedation, 3 planes (transverse, coronal, sagittal) of imaging should be obtained in the post-contrast acquisition. Post-operative imaging in brain tumors should ideally be performed within 24 to $48 \mathrm{hr}$ of the operation. Long-term follow-up imaging relies on the availability of prior imaging studies for comparison, and history regarding treatment protocols.

Proton MR spectroscopy studies have shown that contrast administration will alter spectra by decreasing the peak area of cholinecontaining compounds. Tumors are frequently heterogeneous histologically and placement of the voxel in different regions of the tumor may yield different MR spectroscopy results. The relatively large voxel size also results in volume averaging with the adjacent brain. In routine clinical use, the post-contrast images are used to identify the solid appearing portion of the lesion and the voxel is placed in the solid (enhancing portion) of the lesion (Ricci et al., 2000).

\section{Anticipated Results}

The goal in evaluating post-therapy brain tumors is to image and localize the site of documented tumor and assess for residual/recurrent tumor. The dilemma of distinguishing radiation necrosis from residual/recurrent tumor remains. Correlation with the timing of a given tumor therapy is helpful, however frequently a tissue biopsy is needed to solve the issue.

\section{Time Considerations}

The Basic Protocol 1 should take $\sim 30$ min to complete. Proton MR spectroscopy (see Basic Protocol 2) adds $\sim 10$ to $15 \mathrm{~min}$ to the imaging session.

\section{Literature Cited}

Atlas, S.W. and Lavi, E. 1996. Intra-axial brain tumors. In Magnetic Resonance Imaging of the Brain and Spine, 2nd Edition (S.W. Atlas, ed.) pp. 315-422. Lippincott-Raven, Philadelphia.

Grossman, R.I., Hecht-Leavitt, C.M., Evans, S.M., Lenkinski, R.E., Holland, G.A., Van Winkle, T.J., McGrath, J.T., Curran, W.J., Shetty, A., and Joseph, P.M. 1988. Experimental radiation injury: Combined MR imaging and spectroscopy. Radiology 169:305-309.

Nelson, S.J., Vigneron, D.B., and Dillon, W.P. 1999. Serial evaluation of patients with brain tumors using volume MRI and 3D 1H MRSI. N.M.R. Biomed. 12:123-138.

Ricci, P.E., Pitt, A., Keller, P.J., Coons, S.W., and Heiserman, J.E. 2000. Effect of voxel position on single-voxel MR spectroscopy findings. Am. J. Neuroradiol. 21:367-374.

Shellock, F.G. 1996. Pocket Guide to MR Procedures and Metallic Objects. Lippincott-Raven, Philadelphia.

Sijens, P.E., van den Bent, M.J., Nowak, P.J., van Dijk, P., and Ouderk, M. 1997. ${ }^{1} \mathrm{H}$ chemical shift imaging reveals loss of brain tumor choline signal after administration of Gd-contrast. Magn. Reson. Med. 37:222-225.

Taylor, J.S., Langston, J.W., Reddick, W.E., Wingsley, P.B., Ogg, R.J., Pui, M.H., Kun, L.E., Jenkins, J.J. 3rd, Chen, G., Ochs, J.J., Sanford, R.A., and Heideman, R.L. 1996. Clinical value of proton magnetic resonance spectroscopy for differentiating recurrent or residual brain tumor from delayed cerebral necrosis. Int. J. Radiat. Oncol. Biol. Phys. 36:1251-1261.

Contributed by Annette O. Nusbaum

New York Presbyterian Hospital

New York, New York

Scott W. Atlas

Stanford University Medical Center

Stanford, California
Cerebral Neoplastic Disease

A3.5.7 